\title{
Evaluation of the Frequency Following Response as a Predictive Biomarker of Response to Cognitive Training in Schizophrenia
}

\author{
Peter E. Clayson*1, Juan L. Molina², Yash B. Joshi' ${ }^{2,3}$, Michael L. Thomas ${ }^{4}$, Joyce Sprock ${ }^{3}$, John \\ Nungaray $^{3}$, Neal R. Swerdlow ${ }^{3}$, Gregory A. Light ${ }^{2,3}$ \\ ${ }^{1}$ Department of Psychology, University of South Florida, Tampa, FL, USA \\ ${ }^{2}$ VISN 22 Mental Illness Research, Education, \& Clinical Center (MIRECC), San Diego VA \\ Healthcare System, San Diego, CA, USA \\ ${ }^{3}$ Department of Psychiatry, University of California San Diego, San Diego, CA, USA
}

*Corresponding author at: Department of Psychiatry, University of California San Diego, 9500 Gilman Drive \#0804, La Jolla, CA, USA, 92093. Email: glight@ucsd.edu 


\begin{abstract}
Neurophysiological biomarkers of auditory processing show promise predicting outcomes following auditory-based targeted cognitive training (TCT) interventions in patients with schizophrenia, but the viability of the early frequency following response (FFR) as a biomarker has yet to be examined, despite its ecological and face validity for auditory-based interventions. FFR is an event-related potential (ERP) that reflects the earliest stages of auditory processing. We predicted that schizophrenia patients would show acute- and longer-term FFR malleability in the context of auditory-based TCT. Fifty-two schizophrenia patients were randomized to either TCT $(n=30)$ or treatment as usual (TAU; $n=22)$, and electroencephalography was recorded during rapid presentation of an auditory speech stimulus before treatment, after one hour of training, and after 30 hours of training. Patients in the TCT group did not show changes in FFR after training, but FFR measurements in the TAU group diminished over time. FFR was positively associated with performance on a measure of single word-in-noise perception in the TCT group, and with performance on a measure of sentence-in-noise perception in both TCT and TAU groups. Psychometric reliability analyses of FFR scores indicated high internal consistency but low test-rest reliability between sessions one hour and 12 weeks apart. While the present study did not find evidence of FFR malleability or utility in predicting response to auditorybased TCT, significant associations were observed between FFR measures and behavioral measures of speech discriminability. These findings support the dissociation between measures of speech discriminability along the hierarchy of cortical and subcortical early auditory information processing in schizophrenia.
\end{abstract}

Keywords: frequency following response (FFR), schizophrenia, speech-in-noise recognition, auditory cognitive training, event-related brain potential (ERP), ERP psychometric reliability 


\section{Introduction}

Abnormalities in early auditory information processing are linked to cognitive impairment and psychosocial disability in schizophrenia (Ahmed et al., 2020; Elvevag and Goldberg, 2000; Green et al., 2000; Koshiyama et al., 2021d; Molina et al., 2020; Thomas et al., 2017), and these abnormalities have been documented across stages of illness (Ahmed et al., 2020; Hamilton et al., 2020; Jahshan et al., 2012). This evidence mobilized investigations of electroencephalographic (EEG) biomarkers that index auditory processing for use in procognitive therapeutics, such as "bottom-up" auditory-based targeted cognitive training (TCT) interventions (Light et al., 2020; Swerdlow et al., 2018). Mismatch negativity (MMN), P3a, and auditory steady-state responses (ASSRs) are neurophysiologic biomarkers that are sensitive to or predictive of outcome following TCT (Hochberger et al., 2019; Molina et al., 2020). We sought to determine whether an index of early speech encoding, the frequency-following response (FFR), is similarly acutely sensitive to or predictive of outcomes following TCT. Despite strong ecological and face validity of FFR in the context of auditory-based interventions, to our knowledge this measure has only recently been studied in schizophrenia (Clayson et al., 2020).

FFRs largely represent a manifestation of auditory midbrain activity, which is sensitive to changes in a distributed neural system that supports auditory processing (Coffey et al., 2019). FFR is an event-related brain potential (ERP) that reflects the earliest stages (e.g., within 7 to 9 ms) of sound-evoked neural activity (Kraus and White-Schwoch, 2015, 2017). Other ERP indices of auditory processing linked to clinically relevant outcomes in schizophrenia (e.g., MMN or P3a, see Joshi et al., 2018; Light et al., 2015) represent subsequent stages of cortical processing, 100 to 400 ms later than FFRs (Koshiyama et al., 2021a; Koshiyama et al., 2020a, b; Koshiyama et al., 2021b, c; Rissling et al., 2014; Takahashi et al., 2013). FFRs to speech have 
more complex spectral and temporal architectures than tones, chirps, or clicks, which are used to elicit MMN, P3a, or ASSRs; FFRs represent the neural encoding of acoustic properties of a stimulus (Kraus and White-Schwoch, 2015, 2017). Therefore, if FFRs are sensitive to auditorybased TCT, such findings would indicate that TCT enhances the earliest stages of auditory sensory processing at the level of midbrain (Tarasenko et al., 2014).

Support for the use of FFRs in the context of TCT comes from studies of training interventions outside of schizophrenia (Elmer et al., 2017; Russo et al., 2005; Skoe et al., 2013; Song et al., 2008). In these studies, FFRs were sensitive to short- and long-term interventions in healthy participants and children with language disorders; changes in FFR predicted improvements in cognition. These studies demonstrate that FFR is malleable and predict sensitivity to auditory interventions in certain clinical populations, but the malleability of FFR in schizophrenia has yet to be examined.

We recently demonstrated that schizophrenia patients showed FFR deficits (Clayson et al., 2020). Importantly, some commonly used FFR measurements showed strong psychometric internal consistency, which supports their potential utility as predictive biomarkers of treatment. Therefore, we aimed to assess the malleability of FFRs and their suitability as predictive biomarkers of auditory-based TCT in a cohort of schizophrenia patients. Based on findings of FFR malleability in other populations (e.g., Elmer et al., 2017), we predicted that patients with schizophrenia would show acute- and longer-term FFR malleability in the context of TCT. We also examined relationships between FFR measurements and speech-in-noise perception to determine whether speech-in-noise perception was linked to changes in FFR.

\section{Method}

\subsection{Participants}


Study enrollment included 52 treatment-refractory patients with schizophrenia or schizoaffective disorder. Patients were recruited from a community-based inpatient treatment program as described in our previous studies (Hochberger et al., 2019; Hochberger et al., 2020; Joshi et al., 2019; Molina et al., 2020; Thomas et al., 2018a; Thomas et al., 2018c). This study was approved by the Institutional Review Board of the University of California, San Diego (IRB\#130874). All participants provided written informed consent and subsequent written approvals from public guardians/conservators were also obtained for all patients.

Psychiatric diagnoses were confirmed using the Structured Clinical Interview for the Diagnostic and Statistical Manual of Mental Disorders, Fourth Edition (SCID; First et al., 1996). Exclusion criteria included an inability to understand the consent process and/or provide consent, insufficient English fluency, auditory detection threshold above $40 \mathrm{~dB}$ at $1 \mathrm{kHz}$, history of head injury with loss of consciousness exceeding thirty minutes, neurological illness, severe systemic illness, or current mania.

Patients completed a baseline assessment and then were randomized to either TCT ( $n=$ 30) or treatment-as-usual (TAU; $n=22$ ), using stratified random assignment by discrete levels of age, ethnicity, and gender. Treatment teams were blinded to group assignment, but study staff and participants were not blinded due to the nature of the treatment and resource limitation, such as personnel and space in the research facility. The baseline assessment included clinical symptom ratings, assessments of cognition, and collection of FFRs (see below). Following the baseline assessment, participants completed one hour of TCT or of computer games (TAU). The FFR task was then repeated immediately and at the end of treatment (12-13 weeks after baseline assessment).

\subsection{Speech Perception in Noise}


Measures of speech perception in noise included the Words-in-Noise (WIN) test (Zecker et al., 2013) and the Quick Speech-in-Noise Test (SIN, Killion et al., 2004). Tests were modified to allow for binaural presentation. The WIN tests the ability to recognize single words presented amid varying volume levels of background noise ( $24 \mathrm{~dB}$, easy, to $-2 \mathrm{~dB}$ SNR, difficult). The primary measure of interest was the number of words correctly recalled during the $4 \mathrm{~dB}$ block, because this level maximally differentiates patients and healthy comparison participants

(Swerdlow et al., 2020). During the SIN, participants are tested on their ability to remember five key words of a sentence amid varying volume levels of background noise. Thirty total words are presented across six sentences while speech over background noise decreases $(25 \mathrm{~dB}$, easy, to 0 $\mathrm{dB}$, difficult). The total recalled words were subtracted from 25.5 to convert to an SNR Loss score, which was the primary measure of interest (Wilson et al., 2007).

\subsection{Cognitive and Clinical Assessments}

All participants completed the MATRICS Consensus Cognitive Battery (MCCB) to assess cognitive functioning (Nuechterlein and Green, 2006). The MCCB assessed six different cognitive domains: attention/vigilance, reason and problem solving, speed of processing, working memory, verbal learning, and visual learning. Scores from the MCCB were converted into standardized $T$ scores $(M=50, S D=10)$ and adjusted for age and gender (Kern et al., 2008). Psychiatric symptoms were assessed using the Scale for the Assessment of Positive Symptoms (SAPS) and the Scale for the Assessment of Negative Symptoms (Andreasen, 1984a, b).

\subsection{Experimental FFR Task}

Participants listened to binaurally presented stimuli via insert earphones at $75 \mathrm{~dB}$ SPL while seated comfortably in a reclining chair watching a silent cartoon. Stimuli comprised 6,000 five-formant synthesized speech sounds (speech syllable 'da') presented for a duration of $40 \mathrm{~ms}$ 
with an inter-stimulus interval of 90 ms (see Klatt, 1980; Krizman and Kraus, 2019). The 'da' stimulus was presented in alternating polarity. The fundamental frequency ( $\mathrm{F}_{0}: 103$ to $\left.125 \mathrm{~Hz}\right)$ and first three formants ( $\mathrm{F}_{1}: 220$ to $720 \mathrm{~Hz}, \mathrm{~F}_{2}: 1,700$ to $1,200 \mathrm{~Hz}, \mathrm{~F}_{3}: 2,580$ to $2,500 \mathrm{~Hz}$ ) changed linearly over the course of a stimulus. $\mathrm{F}_{4}$ and $\mathrm{F}_{5}$ remained constant at $3,600 \mathrm{~Hz}$ and 4,500 Hz, respectively (see Krizman et al., 2012).

\subsection{Electrophysiological Data Recording and Reduction}

Continuous EEG was recorded using an ActiveTwo BioSemi amplifier (BioSemi, Amsterdam, Netherlands), which uses active $\mathrm{Ag} / \mathrm{AgCl}$ sintered electrodes. EEG signals were digitized at a sampling rate of $8,192 \mathrm{kHz}$ with a 24-bit analog-to-digital converter (least significant bit: $31.25 \mathrm{nV}$ ). EEG was recorded from 64 active scalp electrodes placed based on the 10/20 system using a custom cap (Cortech Solutions, Wilmington, North Carolina, USA). Two additional scalp electrodes were placed on each mastoid. Electrooculogram (EOG) was recorded from four additional sensors placed above and below the left eye and near the outer canthi. EEG and EOG channels were referenced online to a common mode sense electrode. Lastly, the audio input was digitally encoded on a dedicated A/D channel to ensure accurate timing of stimuluslocked epochs, and event markers were adjusted to the onset of the audio stimulus recorded in the A/D channel.

EEG data were re-refenced to algebraically linked mastoids and filtered using a fourthorder (24 dB/oct) infinite impulse response Butterworth filter with half-amplitude cutoffs at 100 $\mathrm{Hz}$ and 2,000 Hz implemented in ERPLab v8.02 (Lopez-Calderon and Luck, 2014). Stimuluslocked epochs were extracted for each participant using a temporal window of $15 \mathrm{~ms}$ prior to the onset of the audio stimulus to $60 \mathrm{~ms}$ following the onset of the stimulus. Epochs were rejected if a voltage difference of more than $23.8 \mu \mathrm{V}$ was detected at $\mathrm{Cz}$ within any epoch (e.g., Krizman et 
al., 2019; Otto-Meyer et al., 2018). The 15-ms period prior to the onset of the audio stimulus was used for baseline adjustment. All ERP measurements were scored at channel $\mathrm{Cz}$ (Krizman and Kraus, 2019).

\subsection{Broadband Response Magnitude}

We selected four estimates of broadband response magnitude due to their excellent internal consistency ( $\phi s \geq .96$ ) based on our prior study of healthy subjects and this cohort of patients (Clayson et al., 2020). The root mean square (RMS) of amplitude between -15 and $0 \mathrm{~ms}$ prior to presentation of the audio stimulus and between 19.5 and $44.2 \mathrm{~ms}$ following presentation of the stimulus were extracted (for review of measurement approaches, see Krizman and Kraus, 2019). These RMS measurements were then used to create a measure of relative magnitude termed the signal-to-noise ratio (SNR). The SNR is computed by dividing post-stimulus RMS activity by pre-stimulus RMS activity, and can be converted to dB by computing 20 times the base $\log 10$ of this score (i.e., dB SNR).

Psychometric reliability was verified using generalizability theory equations that simultaneously estimate internal consistency and test-retest reliability. Our prior study reported only internal consistency based on single-session data in this cohort (Clayson et al., 2020), and test-retest reliability of FFR scores has not been examined in schizophrenia. Generalizability coefficients of equivalence (internal consistency) and coefficients of stability (test-retest reliability) were estimated (Baldwin et al., 2015; Brennan, 2001; Carbine et al., in press; Clayson et al., 2021b; Clayson et al., in press; Shavelson and Webb, 1991; Vispoel et al., 2018). Reliability for each measurement and group was analyzed using the ERP Reliability Analysis (ERA) Toolbox v 0.5.3 (Clayson et al., 2021a; Clayson et al., in press; Clayson and Miller, 2017). The ERA Toolbox used CmdStan v 2.21.0 (Stan Development Team, 2019) to implement 
the analyses in Stan (Carpenter et al., 2017). Coefficients of equivalence across measures and groups ranged from .96 to $>.99$, and coefficients of stability ranged from .15 to .79 .

\subsection{Data Analysis}

We examined the impact of TCT on FFRs using multilevel models fit in the $R$ package lme4 (Bates et al., 2015). Measurements were nested within participants, and each model included a global intercept, fixed effects estimates (group, time), and random intercepts for participants. Separate models were then run using WIN $4 \mathrm{~dB}$ and SIN as predictors, which permitted the examination of whether speech-in-noise perception related to FFR measurements or to changes in those measurements following TCT and whether groups showed similar bivariate relationships.

All multilevel models used maximum likelihood estimation and Sattherwaite degrees of freedom for testing significant effects. Initial analyses of variances (ANOVAs) on modeled data assessed the statistical significance of higher-order effects to justify the examination of first-

order predictors. For example, coefficients for the main effect of time (baseline [reference level], 1-hr follow up, 12-week follow up) were only interpreted when a statistically significant main effect of time was observed in the overall ANOVA. Tests of estimated marginal means were used to follow up significant tests using the $R$ package emmeans (Lenth, 2020).

\section{Results}

Summary demographic information is shown in Table 1, and summary scores of ERP measurements are shown in Table 2. Grand average waveforms are shown in Figure 1.

\subsection{FFR Malleability}

Multilevel models predicting treatment-related changes in FFR are shown in Table 3, and estimated marginal means are shown in Figure 2. For the model predicting pre-stimulus RMS, 
main effects of group and time failed to reach conventional levels of statistical significance, $F(1$, $48.72)=3.85, p=.06 ; F(2,85.43)=2.37, p=.10$, respectively. The Group $\mathrm{x}$ Time interaction reached significance, $F(2,85.43)=3.31, p=.04$. The TAU group showed larger pre-stimulus RMS at the 12-week follow up than at baseline and at the 1-hour follow up, $b=-.01, t(84.2)=-$ $2.14, p=.04 ; b=-.01, t(84.2)=-2.93, p<.01$, respectively. There was no difference between baseline and the 1 -hour follow up, $b=0.004, t(83.3)=0.82, p=.41$. The TAU group showed larger pre-stimulus RMS than the TCT group at the 12-week follow up, $b=0.02, t(94.0)=2.66$, $p=.01$, but group differences were not observed at baseline or at the 1 -hour follow up $(|t \mathrm{~s}|<1.9$, $p s>.06)$. There were no significant between-session differences within the TCT group $(|t \mathrm{~s}|<1.6$, ps >.13).

Significant effects were not observed for the ANOVA on the model predicting changes in stimulus-related RMS $(F \mathrm{~s}<0.7, p \mathrm{~s}>.40)$.

The ANOVA for SNR yielded nonsignificant main effects $\left(F_{\mathrm{s}}<1.9, p \mathrm{~s}>.17\right)$, and a significant Group x Time interaction, $F(2,84.46)=4.82, p=.01$. The TAU group showed a decrease from the 1-hour to the 12-week follow up, $b=0.64, t(83.3)=2.76, p=.01$, but no other between-session differences were significant $(|t \mathrm{~s}|<1.5, p \mathrm{~s}>.14)$. Between-session changes were not observed for the TCT group $(|t \mathrm{~s}|<1.7, p \mathrm{~s}>$.09). The TCT group showed larger SNR than the TAU group at 12 weeks, $b=-.82, t(90.1)=-2.25, p=.03$, but significant differences were not observed at baseline or the 1-hour follow up $(|t \mathrm{~s}|<1.6, p \mathrm{~s}>.11)$. Therefore, group differences in SNR of FFR at the 12-week follow-up were due to worsening SNR in the TAU group, not improvement in SNR in the TCT group.

The main effects of group and time were not significant for the ANOVA model predicting dB SNR $(F \mathrm{~s}<2.6, p \mathrm{~s}>.11)$, but the Group $\mathrm{x}$ Time interaction was, $F(2,85.45)=$ 
$4.45, p=.01$. The pattern of effects was identical to the SNR analyses. The TAU group showed a decrease in dB SNR from the 1-hour follow up to the 12-week follow up, $b=2.34, t(84.1)=$ $3.27, p<.01$, but no other between-session differences reached conventional levels of significance $(|t \mathrm{~s}|<1.9, p \mathrm{~s}>.06)$. Between-session changes were not significant for the TCT group $(|t s|<1.6, p s>.12)$. dB SNR was larger in the TCT group than in the TAU group at 12 weeks, $b=-2.59, t(96.7)=-2.42, p=.02$, but significant group differences were not observed at baseline or the 1-hour follow up $(|t \mathrm{~s}|<1.8, p \mathrm{~s}>.08)$.

\subsection{Predictors of FFR Malleability}

We next examined whether $4 \mathrm{db}$ WIN scores and SIN performance predicted FFR malleability (see Table 4).

3.2.1. WIN. The ANOVA on the model predicting post-stimulus RMS indicated a main effect of time, $F(2,77.87)=4.94, p=.01$, but the follow-up pairwise contrasts were not significant $(|t \mathrm{~s}|<1.1, p \mathrm{~s}>$.28). Although main effects of group and WIN scores were not significant $(F \mathrm{~s}<3.6, p \mathrm{~s}>.06)$, the Group $\mathrm{x}$ WIN Scores interaction was, $F(1,46.14)=4.29, p=$ .04. The TCT group showed a stronger relationship between WIN scores and post-stimulus RMS, $b=0.02, t(47.4)=2.06, p=.04$, than the TAU group, $b=-.01, t(45.2)=-.96, p=.34$ (see Figure 3). The Time $\mathrm{x}$ WIN Scores interaction was also significant, $F(2,78.00)=4.54, p=.01$, but the follow-up contrasts for the relationship between WIN Scores and post-stimulus RMS at each assessment were not significant, $(|t \mathrm{~s}|<1.2, p \mathrm{~s}>.25)$. None of the remaining interactions were significant $(F \mathrm{~s}<0.4, p \mathrm{~s}>.68)$.

The ANOVAs indicated nonsignificant main effects and interactions for the models predicting pre-stimulus RMS, SNR, and dB SNR $(F \mathrm{~s}<2.6, p \mathrm{~s}>.11)$. 
3.2.2. SIN. For the model predicting pre-stimulus RMS, the main effect of SIN scores was significant, $F(1,53.11)=8.06, p=.01$, with higher SIN scores related to lower pre-stimulus RMS, $b=-.002, t(79.84)=-2.15, p=.03$ (see Figure 3). The remaining main effects and interactions were not significant $(F \mathrm{~s}<1.1, p \mathrm{~s}>.31)$.

An ANOVA on the model predicting dB SNR indicated a significant main effect of SIN scores, $F(1,53.68)=4.66, p=.04$ (see Figure 3), but the individual predictor for SIN scores was not significant, $b=0.25, t(79.63)=1.63, p=.11$. The remaining main effects and interactions were not significant $(F \mathrm{~s}<2.2, p \mathrm{~s}>.11)$.

Main effects and interactions for the ANOVAs predicting stimulus-related RMS and the model predicting SNR failed to reach conventional levels of significance $(F \mathrm{~s}<3.6, p \mathrm{~s}>.06)$.

3.2.3. Exploratory Analyses. We next examined whether other covariates predicted changes in FFR to understand the worsening FFR over time in the TAU group. Separate multilevel models evaluated the impact of anticholinergic burden, chlorpromazine equivalents, age, and total positive or negative symptom scores on FFR. Although there was a Time x Age interaction for post-stimulus RMS, $F(2,79.58)=7.58, p<.001$, the change between baseline and the 12-week follow up was not significant, $t(79.57)=0.36, p=.72$, which suggests that treatment group differences at the 12-week follow up were not explained by the age of participants. None of the remaining interactions with time were significant $(F \mathrm{~s}<2.3, p \mathrm{~s}>.11)$.

\subsection{FFR as a Predictor}

Separate exploratory regression analyses used baseline FFR measurements or FFR change scores (1-hour follow up minus baseline) to predict changes (12-week minus baseline) in total MCCB scores, MCCB subscale scores, and total positive or negative symptoms. Models 
predicted changes in each dependent variable from an FFR measurement, group, and the interaction. None of the models was significant $(F \mathrm{~s}<2.8, p \mathrm{~s}>.05)$.

\section{Discussion}

The malleability of FFR in response to auditory-based TCT was examined in a group of patients with schizophrenia. Although we predicted that patients in the TCT group would show improvements in FFR, statistically significant changes were not observed. Group differences were observed at the 12-week follow up, but these differences were due to FFR measurements in the TAU group unexpectedly worsening over the course of the study. Regarding measures of speech perception in noise, only the TCT group showed significant relationships between poststimulus RMS and WIN performance, such that better performance on the WIN was related to larger post-stimulus RMS. Better performance on the SIN was associated with better FFR measurements across both groups (lower pre-stimulus RMS and higher db SNR).

Unlike studies of other populations (e.g., Elmer et al., 2017), certain FFR measurements were not sensitive to an auditory-based intervention in patients with schizophrenia. The present TCT used a broad training regimen that included discriminating between tones and syllables and an emphasis on auditory memory (Thomas et al., 2018b). Other studies of FFR malleability emphasized phonetic discrimination training that typically consisted of judging whether two syllables were identical (e.g., Elmer et al., 2017). It is possible that phonetic discrimination training is the key intervention to observe FFR malleability. Other EEG measures of auditory processing, such as MMN, P3a, and ASSR, are acutely sensitivity to TCT and predictive of treatment response (e.g., Hochberger et al., 2019). These measures represent activity from a broadly distributed and dynamic network of cortical sources and reflect cortical stages of processing that are slightly later than FFRs. Therefore, FFRs may be more sensitive to specific 
types of "bottom up" training that leverages more proximal neural circuits than those targeted by TCT. In the context of TCT, measures with greater contributions from higher order cortical networks may serve as more sensitive predictive biomarkers of treatment response to TCT in schizophrenia.

Larger post-stimulus RMS related to better performance on the WIN $4 \mathrm{~dB}$ level, which suggests that the patients with sharper sensory representations of the auditory stimulus are those who are likely to recall single words in the presence of background noise. The WIN $4 \mathrm{~dB}$ level is also the performance level most sensitive to memantine- or TCT-based enhancements of auditory information processing (Molina et al., under review; Swerdlow et al., 2020). In light of the group differences in correlations for the WIN and similar relationships across groups for SIN, present findings provide further evidence that WIN and SIN represent partially distinct aspects of auditory processing.

Unlike WIN performance, both groups showed better SIN performance related to lower pre-stimulus RMS. Higher auditory processing fidelity (i.e., lower pre-stimulus RMS) suggests that the auditory system is suppressing task-irrelevant inputs, which was likely reflected in better discrimination of sentences from background noise. Higher SNR generally reflects higher phaselocking accuracy of auditory nerve fibers (Carcagno and Plack, 2017). SNR is the ratio of poststimulus RMS (i.e., signal) to pre-stimulus RMS (i.e., background noise) and indexes the sustained response to the stimulus. SNR tends to be low when participants hear an auditory stimulus in the presence of background noise compared to a stimulus without noise, which indicates SNR is sensitive to noise that hinders auditory discrimination (Prévost et al., 2013; Russo et al., 2004). Therefore, the relationship between dB SNR and SIN performance suggests 
that phase locking of auditory nerve fibers relates to better auditory discriminability of sentences amid ambient background noise.

Present analyses indicated that some FFR measurements worsened in the TAU group. The present cohort was receiving high doses of antipsychotic medications with a high anticholinergic burden. Our group reported verbal learning deficits related to anticholinergic burden during TAU, but not TCT (Joshi et al., 2019). This differential association contributed to speculation that TCT blunts the deleterious impact of anticholinergic burden. In the present analyses, anticholinergic burden and chlorpromazine equivalents did not relate to decreases in FFR during TAU. Other findings indicate that TCT vs. TAU may have neuroprotective, rather than neurorestorative, effects in early schizophrenia (Ramsay et al., 2021), which offers another potential explanation of the worsening FFR during TAU. As this is the first study of FFR in the context of an intervention trial in schizophrenia, further studies are needed to understand the longitudinal dynamics of FFR in patients.

This study has some limitations. First, the present sample included treatment refractory patients who were chronically impaired and in long-term residential care. It is possible that treatment effects were limited by the significant disability in this cohort. However, previous analyses of this cohort indicated that these patients still benefitted clinically from TCT (Hochberger et al., 2019; Thomas et al., 2018b). Second, the present study used standard EEG equipment and data collection protocols. Equipment optimized for recording brainstem responses may yield higher quality estimates, although present FFR measurements yielded high internal consistency. Third, there is no consensus on the optimal approach for measuring FFR (Krizman and Kraus, 2019), and commonly used measurements would benefit from further development (Clayson et al., 2020). Fourth, groups differed in baseline FFR. Although a stratified 
randomization matrix was used to assign patients to groups based on demographics, FFR was not. Given the complexity of processing and managing FFR, stratified random assignment using FFR is not feasible for real-world trials.

In conclusion, FFR measurements did not show acute- or longer-term malleability or predict a beneficial response to auditory-based TCT. Baseline measures of speech perception in noise were positively related to post-stimulus RMS only in the TCT group, which provides evidence for a dissociation between WIN and SIN along the hierarchy of cortical and subcortical early auditory processing. This study complements work using cortical EEG biomarkers, such as MMN, P3a, and ASSRs, as predictors of TCT-related outcomes; compared to FFR, these other EEG biomarkers appear to be better predictors of pro-cognitive response in schizophrenia. 


\section{References}

Ahmed, S., Lepock, J.R., Mizrahi, R., Bagby, R.M., Gerritsen, C.J., Korostil, M., Light, G.A., Kiang, M., 2020. Decreased Gamma Auditory Steady-State Response Is Associated With Impaired Real-World Functioning in Unmedicated Patients at Clinical High Risk for Psychosis. Clin EEG Neurosci, 1550059420982706.

Altman, D.G., Dore, C.J., 1990. Randomisation and baseline comparisons in clinical trials. Lancet 335(8682), 149-153.

Andreasen, N.C., 1984a. Scale for the Assessment of Negative Symptoms (SANS). University of Iowa, Iowa City, IA.

Andreasen, N.C., 1984b. Scale for the Assessment of Positive Symptoms (SAPS). University of Iowa, Iowa City, IA.

Baldwin, S.A., Larson, M.J., Clayson, P.E., 2015. The dependability of electrophysiological measurements of performance monitoring in a clinical sample: A generalizability and decision analysis of the ERN and Pe. Psychophysiology 52(6), 790-800.

Bates, D., Mächler, M., Bolker, B., Walker, S., 2015. Fitting linear mixed-effects models using lme4. Journal of Statistical Software 67(1), 1-48.

Begg, C.B., 1990. Suspended judgment. Significance tests of covariate imbalance in clinical trials. Control Clin Trials 11(4), 223-225.

Brennan, R.L., 2001. Generalizability theory: Statistics for social science and public policy. Springer-Verlag, New York, NY.

Carbine, K.A., Clayson, P.E., Baldwin, S.A., LeCheminant, J., Larson, M.J., in press. Using generalizability theory and the ERP Reliability Analysis (ERA) Toolbox for assessing test-retest reliability of ERP scores Part 2: Application to food-based tasks and stimuli. International Journal of Psychophysiology.

Carcagno, S., Plack, C.J., 2017. Short-term learning and memory: Training and perceptual learning, in: Kraus, N., Anderson, S., White-Schwoch, T., Fay, R.R., Popper, A.N. (Eds.), The frequency-following response: A window into human communication. Springer International Publishing, Cham, pp. 75-100.

Carpenter, B., Gelman, A., Hoffman, M., Lee, D., Goodrich, B., Betancourt, M., Brubaker, M.A., Guo, J., Li, P., Riddell, A., 2017. Stan: A probabilistic programming language. Journal of Statistical Software 76(1).

Clayson, P.E., Baldwin, S.A., Larson, M.J., 2021a. Evaluating the internal consistency of subtraction-based and residualized difference scores: Considerations for psychometric reliability analyses of event-related potentials. Psychophysiology 58(4), e13762.

Clayson, P.E., Brush, C.J., Hajcak, G., 2021b. Data quality and reliability metrics for eventrelated potentials (ERPs): The utility of subject-level reliability. International Journal of Psychophysiology 165, 121-136.

Clayson, P.E., Carbine, K.A., Baldwin, S.A., Olsen, J.A., Larson, M.J., in press. Using generalizability theory and the ERP Reliability Analysis (ERA) Toolbox for assessing test-retest reliability of ERP scores Part 1: Algorithms, framework, and implementation. International Journal of Psychophysiology.

Clayson, P.E., Joshi, Y.B., Thomas, M.L., Tarasenko, M., Bismark, A., Sprock, J., Nungaray, J., Cardoso, L., Wynn, J.K., Swerdlow, N., Light, G.A., 2020. The viability of the frequency following response characteristics for use as biomarkers of cognitive therapeutics in schizophrenia. PsyArXiv. 
Clayson, P.E., Miller, G.A., 2017. ERP Reliability Analysis (ERA) Toolbox: An open-source toolbox for analyzing the reliability of event-related potentials. International Journal of Psychophysiology 111, 68-79.

Coffey, E.B.J., Nicol, T., White-Schwoch, T., Chandrasekaran, B., Krizman, J., Skoe, E., Zatorre, R.J., Kraus, N., 2019. Evolving perspectives on the sources of the frequency-following response. Nature Communications 10(1), 5036-5010.

Elmer, S., Hausheer, M., Albrecht, J., Kühnis, J., 2017. Human brainstem exhibits higher sensitivity and specificity than auditory-related cortex to short-term phonetic discrimination learning. Scientific Reports 7(1), 7455.

Elvevag, B., Goldberg, T.E., 2000. Cognitive impairment in schizophrenia is the core of the disorder. Critical Reviews in Neurobiology 14(1), 1-21.

First, M.B., Spitzer, R.L., Gibbon, M., Williams, J., 1996. Structured clinical interview for DSMIV Axis I Disorders - Patient Edition (SCID-I/P, Verison 2.0). Biometrics Research Department, New York State Psychiatric Institute, New York.

Green, M.F., Kern, R.S., Braff, D.L., Mintz, J., 2000. Neurocognitive deficits and functional outcome in schizophrenia: Are we measuring the "right stuff"? Schizophrenia Bulletin 26(1), 119-136.

Hamilton, H.K., Boos, A.K., Mathalon, D.H., 2020. Electroencephalography and event-related potential biomarkers in individuals at clinical high risk for psychosis. Biological psychiatry 88(4), 294-303.

Hochberger, W.C., Joshi, Y.B., Thomas, M.L., Zhang, W., Bismark, A.W., Treichler, E.B.H., Tarasenko, M., Nungaray, J., Sprock, J., Cardoso, L., Swerdlow, N., Light, G.A., 2019. Neurophysiologic measures of target engagement predict response to auditory-based cognitive training in treatment refractory schizophrenia. Neuropsychopharmacol 44(3), 606-612. Hochberger, W.C., Thomas, M.L., Joshi, Y.B., Molina, J., Treichler, E.B.H., Nungaray, J., Cardoso, L., Sprock, J., Swerdlow, N., Light, G.A., 2020. Oscillatory biomarkers of early auditory information processing predict cognitive gains following targeted cognitive training in schizophrenia patients. Schizophr Res 215, 97-104.

Jahshan, C., Cadenhead, K.S., Rissling, A.J., Kirihara, K., Braff, D.L., Light, G.A., 2012. Automatic sensory information processing abnormalities across the illness course of schizophrenia. Psychol Med 42(1), 85-97.

Joshi, Y.B., Breitenstein, B., Tarasenko, M., Thomas, M.L., Chang, W.-L., Sprock, J., Sharp, R.F., Light, G.A., 2018. Mismatch negativity impairment is associated with deficits in identifying real-world environmental sounds in schizophrenia. Schizophrenia Research 191, 5-9. Joshi, Y.B., Thomas, M.L., Hochberger, W.C., Bismark, A.W., Treichler, E.B.H., Molina, J., Nungaray, J., Cardoso, L., Sprock, J., Swerdlow, N.R., Light, G.A., 2019. Verbal learning deficits associated with increased anticholinergic burden are attenuated with targeted cognitive training in treatment refractory schizophrenia patients. Schizophrenia Research 208(C), 384-389. Kern, R.S., Nuechterlein, K.H., Green, M.F., Laade, L.E., Fenton, W.S., Gold, J.M., Keefe, R.S.E., Mesholam-Gately, R., Mintz, J., Seidman, L.J., Stover, E., Marder, S.R., 2008. The MATRICS consensus cognitive battery, part 2: Co-norming and standardization. The American Journal of Psychiatry 165(2), 214-220.

Killion, M.C., Niquette, P.A., Gudmundsen, G.I., Revit, L.J., Banerjee, S., 2004. Development of a quick speech-in-noise test for measuring signal-to-noise ratio loss in normal-hearing and hearing-impaired listeners. Journal of the Acoustical Society of America 116(4 I), 2395-2405. 
Klatt, D.H., 1980. Software for a cascade/parallel formant synthesizer. The Journal of the Acoustical Society of America 67(3), 971-995.

Koshiyama, D., Miyakoshi, M., Joshi, Y.B., Molina, J.L., Tanaka-Koshiyama, K., Joyce, S., Braff, D.L., Swerdlow, N.R., Light, G.A., 2021a. Neural network dynamics underlying gamma synchronization deficits in schizophrenia. Prog Neuropsychopharmacol Biol Psychiatry 107, 110224.

Koshiyama, D., Miyakoshi, M., Joshi, Y.B., Molina, J.L., Tanaka-Koshiyama, K., Sprock, J., Braff, D.L., Swerdlow, N.R., Light, G.A., 2020a. Abnormal Effective Connectivity Underlying Auditory Mismatch Negativity Impairments in Schizophrenia. Biol Psychiatry Cogn Neurosci Neuroimaging 5(11), 1028-1039.

Koshiyama, D., Miyakoshi, M., Joshi, Y.B., Molina, J.L., Tanaka-Koshiyama, K., Sprock, J., Braff, D.L., Swerdlow, N.R., Light, G.A., 2020b. A distributed frontotemporal network underlies gamma-band synchronization impairments in schizophrenia patients. Neuropsychopharmacol 45(13), 2198-2206.

Koshiyama, D., Miyakoshi, M., Joshi, Y.B., Nakanishi, M., Tanaka-Koshiyama, K., Sprock, J., Light, G.A., 2021b. Source decomposition of the frontocentral auditory steady-state gamma band response in schizophrenia patients and healthy subjects. Psychiatry Clin Neurosci.

Koshiyama, D., Miyakoshi, M., Joshi, Y.B., Nakanishi, M., Tanaka-Koshiyama, K., Sprock, J., Light, G.A., 2021c. Sources of the frontocentral mismatch negativity and P3a responses in schizophrenia patients and healthy comparison subjects. Int J Psychophysiol 161, 76-85.

Koshiyama, D., Thomas, M.L., Miyakoshi, M., Joshi, Y.B., Molina, J.L., Tanaka-Koshiyama, K., Sprock, J., Braff, D.L., Swerdlow, N.R., Light, G.A., 2021d. Hierarchical Pathways from Sensory Processing to Cognitive, Clinical, and Functional Impairments in Schizophrenia.

Schizophr Bull 47(2), 373-385.

Kraus, N., White-Schwoch, T., 2015. Unraveling the biology of auditory learning: A cognitivesensorimotor-reward framework. Trends in Cognitive Sciences 19(11), 642-654.

Kraus, N., White-Schwoch, T., 2017. Neurobiology of everyday communication: What have we learned from music? The Neuroscientist 23(3), 287-298.

Krizman, J., Bonacina, S., Kraus, N., 2019. Sex differences in subcortical auditory processing emerge across development. Hearing Research 380, 166-174.

Krizman, J., Kraus, N., 2019. Analyzing the FFR: A tutorial for decoding the richness of auditory function. Hearing Research 382, 107779-107716.

Krizman, J., Skoe, E., Kraus, N., 2012. Sex differences in auditory subcortical function. Clinical Neurophysiology 123(3), 590-597.

Lenth, R., 2020. emmeans: Estimated marginal means, aka least-squares means, R package version $1.4 .8 \mathrm{ed}$.

Light, G.A., Joshi, Y.B., Molina, J.L., Bhakta, S.G., Nungaray, J.A., Cardoso, L., Kotz, J.E., Thomas, M.L., Swerdlow, N.R., 2020. Neurophysiological biomarkers for schizophrenia therapeutics. Biomarkers in Neuropsychiatry 2, 100012.

Light, G.A., Swerdlow, N.R., Thomas, M.L., Calkins, M.E., Green, M.F., Greenwood, T.A., Gur, R.E., Gur, R.C., Lazzeroni, L.C., Nuechterlein, K.H., Pela, M., Radant, A.D., Seidman, L.J., Sharp, R.F., Siever, L.J., Silverman, J.M., Sprock, J., Stone, W.S., Sugar, C.A., Tsuang, D.W., Tsuang, M.T., Braff, D.L., Turetsky, B.I., 2015. Validation of mismatch negativity and P3a for use in multi-site studies of schizophrenia: Characterization of demographic, clinical, cognitive, and functional correlates in COGS-2. Schizophrenia Research 163(1-3), 63-72. 
Lopez-Calderon, J., Luck, S.J., 2014. ERPLAB: An open-source toolbox for the analysis of event-related potentials. Frontiers in Human Neuroscience 8(e3004), 213.

Molina, J.L., Joshi, Y.B., Nungaray, J.A., Thomas, M.L., Sprock, J., Clayson, P.E., Sanchez, V., Attarha, M., Biagianti, B., Swerdlow, N., Light, G.A., under review. Central auditory processing deficits in schizophrenia: Effects of auditory-based cognitive training.

Molina, J.L., Thomas, M.L., Joshi, Y.B., Hochberger, W.C., Koshiyama, D., Nungaray, J.A., Cardoso, L., Sprock, J., Braff, D.L., Swerdlow, N.R., Light, G.A., 2020. Gamma oscillations predict pro-cognitive and clinical response to auditory-based cognitive training in schizophrenia. Transl Psychiatry 10(1), 405.

Nuechterlein, K.H., Green, M.F., 2006. MATRICS Consensus Battery. MATRICS Assessment, Inc, Los Angeles, CA.

Otto-Meyer, S., Krizman, J., White-Schwoch, T., Kraus, N., 2018. Children with autism spectrum disorder have unstable neural responses to sound. Experimental Brain Research 236(3), 733-743.

Prévost, F., Laroche, M., Marcoux, A.M., Dajani, H.R., 2013. Objective measurement of physiological signal-to-noise gain in the brainstem response to a synthetic vowel. Clinical Neurophysiology 124(1), 52-60.

Ramsay, I.S., Fryer, S., Roach, B.J., Boos, A., Fisher, M., Loewy, R., Ford, J.M., Vinogradov, S., Mathalon, D.H., 2021. Response to targeted cognitive training may be neuroprotective in patients with early schizophrenia. Psychiatry Research: Neuroimaging 312, 111285.

Rissling, A.J., Miyakoshi, M., Sugar, C.A., Braff, D.L., Makeig, S., Light, G.A., 2014. Cortical substrates and functional correlates of auditory deviance processing deficits in schizophrenia. NeuroImage: Clinical 6, 424-437.

Russo, N., Nicol, T., Musacchia, G., Kraus, N., 2004. Brainstem responses to speech syllables. Clinical Neurophysiology 115(9), 2021-2030.

Russo, N.M., Nicol, T.G., Zecker, S.G., Hayes, E.A., Kraus, N., 2005. Auditory training improves neural timing in the human brainstem. Behavioural Brain Research 156(1), 95-103.

Senn, S., 1991. Baseline comparisons in randomized clinical trials. Stat Med 10(7), 1157-1159.

Senn, S., 1994. Testing for baseline balance in clinical trials. Stat Med 13(17), 1715-1726.

Shavelson, R.J., Webb, N.M., 1991. Generalizability theory: A primer. SAGE Publications, Inc., Thousand Oaks, CA.

Skoe, E., Krizman, J., Spitzer, E., Kraus, N., 2013. The auditory brainstem is a barometer of rapid auditory learning. Neuroscience 243(C), 104-114.

Song, J.H., Skoe, E., Wong, P.C.M., Kraus, N., 2008. Plasticity in the adult human auditory brainstem following short-term linguistic training. Journal of Cognitive Neuroscience 20(10), 1892-1902.

Stan Development Team, 2019. CmdStan: The command-line interface to Stan, version 2.21.0. Swerdlow, N.R., Bhakta, S.G., Light, G.A., 2018. Room to move: Plasticity in early auditory information processing and auditory learning in schizophrenia revealed by acute pharmacological challenge. Schizophrenia Research 199, 285-291.

Swerdlow, N.R., Bhakta, S.G., Talledo, J., Kotz, J., Roberts, B.Z., Clifford, R.E., Thomas, M.L., Joshi, Y.B., Molina, J.L., Light, G.A., 2020. Memantine effects on auditory discrimination and training in schizophrenia patients. Neuropsychopharmacology 45(13), 2180-2188.

Takahashi, H., Rissling, A.J., Pascual-Marqui, R., Kirihara, K., Pela, M., Sprock, J., Braff, D.L., Light, G.A., 2013. Neural substrates of normal and impaired preattentive sensory discrimination 
in large cohorts of nonpsychiatric subjects and schizophrenia patients as indexed by MMN and P3a change detection responses. NeuroImage 66, 594-603.

Tarasenko, M.A., Swerdlow, N.R., Makeig, S., Braff, D.L., Light, G.A., 2014. The auditory brain-stem response to complex sounds: A potential biomarker for guiding treatment of psychosis. Frontiers in Psychiatry 5(OCT), 142.

Thomas, M.L., Bismark, A.W., Joshi, Y.B., Tarasenko, M., Treichler, E.B.H., Hochberger, W.C., Zhang, W., Nungaray, J., Sprock, J., Cardoso, L., Tiernan, K., Attarha, M., Braff, D.L., Vinogradov, S., Swerdlow, N., Light, G.A., 2018a. Targeted cognitive training improves auditory and verbal outcomes among treatment refractory schizophrenia patients mandated to residential care. Schizophr Res 202, 378-384.

Thomas, M.L., Bismark, A.W., Joshi, Y.B., Tarasenko, M., Treichler, E.B.H., Hochberger, W.C., Zhang, W., Nungaray, J., Sprock, J., Cardoso, L., Tiernan, K., Attarha, M., Braff, D.L., Vinogradov, S., Swerdlow, N., Light, G.A., 2018b. Targeted cognitive training improves auditory and verbal outcomes among treatment refractory schizophrenia patients mandated to residential care. Schizophrenia Research 202, 378-384.

Thomas, M.L., Green, M.F., Hellemann, G., Sugar, C.A., Tarasenko, M., Calkins, M.E., Greenwood, T.A., Gur, R.E., Gur, R.C., Lazzeroni, L.C., Nuechterlein, K.H., Radant, A.D., Seidman, L.J., Shiluk, A.L., Siever, L.J., Silverman, J.M., Sprock, J., Stone, W.S., Swerdlow, N.R., Tsuang, D.W., Tsuang, M.T., Turetsky, B.I., Braff, D.L., Light, G.A., 2017. Modeling Deficits From Early Auditory Information Processing to Psychosocial Functioning in Schizophrenia. JAMA Psychiatry 74(1), 37-46.

Thomas, M.L., Treichler, E.B.H., Bismark, A., Shiluk, A.L., Tarasenko, M., Zhang, W., Joshi, Y.B., Sprock, J., Cardoso, L., Tiernan, K., Light, G.A., 2018c. Computerized cognitive training is associated with improved psychosocial treatment engagement in schizophrenia. Schizophr Res 202, 341-346.

Vispoel, W.P., Morris, C.A., Kilinc, M., 2018. Applications of generalizability theory and their relations to classical test theory and structural equation modeling. Psychological Methods 23(1), $1-26$.

Wilson, R.H., McArdle, R.A., Smith, S.L., 2007. An evaluation of the BKB-SIN, HINT, QuickSIN, and WIN materials on listeners with normal hearing and listeners with hearing loss. Journal of Speech, Language, and Hearing Research 50(4), 844-856.

Woods, S.W., 2003. Chlorpromazine equivalent doses for the newer atypical antipsychotics. The Journal of Clinical Psychiatry 64(6), 663-667.

Zecker, S.G., Hoffman, H.J., Frisina, R., Dubno, J.R., Dhar, S., Wallhagen, M., Kraus, N., Griffith, J.W., Walton, J.P., Eddins, D.A., Newman, C., Victorson, D., Warrier, C.M., Wilson, R.H., 2013. Audition assessment using the NIH Toolbox. Neurology 80(11 Suppl 3), S45-48. 
Table 1

Summary of Demographics and Clinical Symptoms

\begin{tabular}{|c|c|c|c|c|}
\hline Characteristic & \multicolumn{2}{|c|}{$\begin{array}{c}\text { Treatment as } \\
\text { Usual }\end{array}$} & \multicolumn{2}{|c|}{$\begin{array}{c}\text { Targeted Cognitiv } \\
\text { Training }\end{array}$} \\
\hline \multirow{3}{*}{ Female/Male } & \multicolumn{2}{|c|}{$n=22$} & \multicolumn{2}{|c|}{$n=30$} \\
\hline & \multicolumn{2}{|c|}{$12 \frac{n}{10}$} & \multicolumn{2}{|c|}{$\stackrel{n}{15 / 15}$} \\
\hline & Mean & $\underline{S D}$ & Mean & $\underline{S D}$ \\
\hline Age (yrs) & 34.3 & $\overline{11.8}$ & 33.9 & $\overline{11.5}$ \\
\hline Education (yrs.) & 11.6 & 2.1 & 11.8 & 1.9 \\
\hline Parental Education (yrs. $)^{1}$ & 12.6 & 4.1 & 13.7 & 3.7 \\
\hline WRAT & 92.5 & 13.4 & 91.4 & 13.6 \\
\hline Quick Speech-in-Noise & 22.9 & 5.0 & 24.0 & 2.6 \\
\hline Words-in-Noise $4 \mathrm{~dB}^{2}$ & 2.3 & 1.2 & 1.7 & 1.3 \\
\hline Duration of illness (years) ${ }^{3}$ & 13.2 & 10.9 & 16.3 & 12.9 \\
\hline Chlorpromazine equivalents (mg/day) & 992 & 777 & 1,329 & 973 \\
\hline Participants with FFR measurements & \multicolumn{2}{|c|}{$\underline{n}$} & \multicolumn{2}{|c|}{$\underline{n}$} \\
\hline Baseline & \multicolumn{2}{|c|}{22} & \multicolumn{2}{|c|}{27} \\
\hline 1-hr follow up & \multicolumn{2}{|c|}{22} & \multicolumn{2}{|c|}{28} \\
\hline 12-week follow up & \multicolumn{2}{|c|}{20} & \multicolumn{2}{|c|}{21} \\
\hline \multicolumn{5}{|l|}{ MCCB } \\
\hline Attention/Vigilance & 29.2 & 15.1 & 31.9 & 10.9 \\
\hline Reason and Problem Solving & 39.3 & 9.8 & 41.3 & 8.6 \\
\hline Speed of Processing & 26.6 & 13.3 & 29.7 & 12.9 \\
\hline Working Memory & 31.1 & 15.0 & 35.0 & 13.2 \\
\hline Verbal Learning & 32.7 & 6.0 & 33.4 & 5.6 \\
\hline Visual Learning & 31.5 & 11.2 & 31.8 & 11.2 \\
\hline Neurocognitive Composite & 22.7 & 14.0 & 25.4 & 11.7 \\
\hline \multicolumn{5}{|l|}{ Symptoms } \\
\hline SANS Global Score & 13.5 & 3.8 & 13.6 & 4.3 \\
\hline SAPS Global Score & 5.5 & 5.0 & 7.2 & 4.7 \\
\hline
\end{tabular}

Note: Consistent with best practice recommendations for clinical trials, groups were not tested for baseline balance (Altman and Dore, 1990; Begg, 1990; Senn, 1991, 1994). Group differences were not observed in subjects who dropped out of the study, $\mathrm{X}^{2}(2)=0.23, p=.89$.

Chlorpromazine equivalents were calculated using formulas from Woods (2003) and summarize information for the 20 patients in the treatment as usual (TAU) group and 23 patients in the targeted cognitive training (TCT) group who were taking medication at the time of testing. ${ }^{1}$ Information is missing for two patients in the TAU group and three patients in the TCT group. ${ }^{2}$ Information is missing for one patient in the TCT group. ${ }^{3}$ Information is missing for two 
patients in the TCT group. SANS = Scales for the Assessment of Negative Symptoms; SAPS = Scales for the Assessment of Positive Symptoms 
Table 2

Summary Event-Related Potential (ERP) Amplitudes ( $\mu V)$ as a Function of Time and Group

\begin{tabular}{|c|c|c|c|c|}
\hline & & Baseline & $\begin{array}{c}\text { 1-Hr } \\
\text { Follow Up }\end{array}$ & $\begin{array}{c}\text { 12-Week } \\
\text { Follow Up }\end{array}$ \\
\hline Group & & $M(S D)$ & $M(S D)$ & $M(S D)$ \\
\hline \multirow[t]{4}{*}{ Treatment as Usual } & Pre-stimulus RMS & $0.06(0.03)$ & $0.05(0.02)$ & $0.07(0.03)$ \\
\hline & Post-stimulus RMS & $0.13(0.05)$ & $0.14(0.08)$ & $0.13(0.04)$ \\
\hline & SNR & $2.62(1.16)$ & $2.95(1.34)$ & $2.22(0.99)$ \\
\hline & dB SNR & $7.67(3.47)$ & $8.67(3.56)$ & $6.14(3.70)$ \\
\hline \multirow{4}{*}{$\begin{array}{l}\text { Targeted Cognitive } \\
\text { Training }\end{array}$} & Pre-stimulus RMS & $0.04(0.02)$ & $0.05(0.03)$ & $0.04(0.02)$ \\
\hline & Post-stimulus RMS & $0.13(0.07)$ & $0.13(0.05)$ & $0.12(0.04)$ \\
\hline & SNR & $3.15(1.02)$ & $2.82(1.08)$ & $3.16(1.60)$ \\
\hline & dB SNR & $9.46(3.16)$ & $8.41(3.25)$ & $9.01(4.23)$ \\
\hline
\end{tabular}

Note: $\mathrm{RMS}=$ root mean square; $\mathrm{SNR}=$ signal-to-noise ratio; $\mathrm{dB}=$ decibel 
Table 3

Estimates from Multilevel Models Predicting Treatment-Related Changes in FFR

\begin{tabular}{|c|c|c|c|c|c|c|c|c|c|c|c|c|}
\hline \multirow[b]{2}{*}{ Parameter } & \multicolumn{3}{|c|}{ Pre-Stimulus RMS } & \multicolumn{3}{|c|}{ Post-Stimulus RMS } & \multicolumn{3}{|c|}{ SNR } & \multicolumn{3}{|c|}{ dB SNR } \\
\hline & $\mathrm{b}$ & $S E$ & $t$ & $\mathrm{~b}$ & $S E$ & $t$ & $\mathrm{~b}$ & $S E$ & $t$ & $\mathrm{~b}$ & $S E$ & $t$ \\
\hline Intercept & 0.055 & 0.005 & $10.64 *$ & 0.129 & 0.013 & $9.81 *$ & 2.620 & 0.262 & $9.99 *$ & 7.672 & 0.762 & $10.07 *$ \\
\hline TCT Group & -0.013 & 0.007 & -1.86 & -0.001 & 0.017 & -0.08 & 0.550 & 0.349 & 1.57 & 1.757 & 1.017 & 1.73 \\
\hline 1-Hr Follow Up & -0.004 & 0.005 & -0.82 & 0.013 & 0.008 & 1.69 & 0.332 & 0.223 & 1.49 & 1.001 & 0.692 & 1.45 \\
\hline 12-Week Follow Up & 0.010 & 0.005 & $2.14 *$ & 0.005 & 0.008 & 0.61 & -0.307 & 0.231 & -1.33 & -1.340 & 0.715 & -1.87 \\
\hline TCT $\times 1-\mathrm{Hr}$ & 0.010 & 0.006 & 1.62 & -0.014 & 0.010 & -1.34 & -0.672 & 0.303 & $-2.22 *$ & -1.982 & 0.936 & $-2.12 *$ \\
\hline TCT x 12-Week & -0.006 & 0.007 & -0.98 & -0.006 & 0.011 & -0.55 & 0.272 & 0.324 & 0.84 & 0.835 & 1.002 & 0.83 \\
\hline
\end{tabular}

Note $:$ RMS = root mean square; $\mathrm{SNR}=$ signal-to-noise ratio; $\mathrm{dB}=$ decibel 
Table 4

Estimates from Multilevel Models Predicting Treatment-Related Changes in FFR from the 4 dB Level of the Words-in-Noise Test (WIN)

\begin{tabular}{lccccccccccccccc}
\hline & \multicolumn{3}{c}{ Pre-Stimulus RMS } & \multicolumn{2}{c}{ Post-Stimulus RMS } & \multicolumn{3}{c}{ SNR } \\
Parameter & $\mathrm{b}$ & $S E$ & $t$ & $\mathrm{~b}$ & $S E$ & $t$ & $\mathrm{~b}$ & $S E$ & \multicolumn{2}{c}{$\mathrm{dB}$ SNR } \\
\hline Intercept & 0.065 & 0.011 & $5.79^{*}$ & 0.146 & 0.027 & $5.32^{*}$ & 2.807 & 0.577 & $4.87 *$ & 7.369 & 1.681 & $4.39^{*}$ \\
TCT Group & -0.029 & 0.014 & -2.14 & -0.059 & 0.033 & -1.78 & 0.222 & 0.699 & 0.32 & 1.693 & 2.039 & 0.83 \\
1-Hr Follow Up & -0.007 & 0.010 & -0.67 & 0.033 & 0.016 & $2.07 *$ & 0.587 & 0.490 & 1.20 & 1.895 & 1.507 & 1.26 \\
12-Week Follow Up & -0.011 & 0.011 & -1.03 & -0.003 & 0.017 & -0.15 & -0.049 & 0.528 & -0.09 & 0.290 & 1.622 & 0.18 \\
WIN & -0.004 & 0.004 & -1.03 & -0.008 & 0.010 & -0.73 & -0.081 & 0.220 & -0.37 & 0.131 & 0.641 & 0.20 \\
TCT x 1-Hr & 0.013 & 0.012 & 1.07 & -0.009 & 0.019 & -0.48 & -0.823 & 0.603 & -1.36 & -2.458 & 1.854 & -1.33 \\
TCT x 12-Week & 0.017 & 0.013 & 1.29 & 0.008 & 0.022 & 0.37 & -0.268 & 0.674 & -0.40 & -1.708 & 2.068 & -0.83 \\
Group x WIN & 0.008 & 0.006 & 1.45 & 0.031 & 0.014 & $2.27 *$ & 0.148 & 0.289 & 0.51 & 0.034 & 0.844 & 0.04 \\
1-Hr x WIN & 0.001 & 0.004 & 0.33 & -0.009 & 0.006 & -1.42 & -0.110 & 0.187 & -0.59 & -0.385 & 0.575 & -0.67 \\
12-Week x WIN & 0.009 & 0.004 & $2.21^{*}$ & 0.003 & 0.006 & 0.45 & -0.106 & 0.196 & -0.54 & -0.674 & 0.601 & -1.12 \\
TCT x 1-Hr x WIN & -0.001 & 0.005 & -0.23 & -0.005 & 0.008 & -0.65 & 0.059 & 0.249 & 0.24 & 0.164 & 0.766 & 0.21 \\
TCT x 12-Week x WIN & -0.010 & 0.006 & -1.83 & -0.007 & 0.009 & -0.80 & 0.284 & 0.284 & 1.00 & 1.222 & 0.871 & 1.40 \\
\hline
\end{tabular}

Note $: \mathrm{RMS}=$ root mean square; $\mathrm{SNR}=$ signal-to-noise ratio; $\mathrm{dB}=$ decibel 
Table 5

Estimates from Multilevel Models Predicting Treatment-Related Changes in FFR from the Quick Speech-in-Noise Test (SIN)

\begin{tabular}{|c|c|c|c|c|c|c|c|c|c|c|c|c|}
\hline \multirow[b]{2}{*}{ Parameter } & \multicolumn{3}{|c|}{ Pre-Stimulus RMS } & \multicolumn{3}{|c|}{ Post-Stimulus RMS } & \multicolumn{3}{|c|}{ SNR } & \multicolumn{3}{|c|}{ dB SNR } \\
\hline & $\mathrm{b}$ & $S E$ & $t$ & $\mathrm{~b}$ & $S E$ & $t$ & $\mathrm{~b}$ & $S E$ & $t$ & $\mathrm{~b}$ & $S E$ & $t$ \\
\hline Intercept & 0.106 & 0.024 & $4.40 *$ & 0.139 & 0.063 & $2.21 *$ & 0.844 & 1.256 & 0.67 & 1.914 & 3.617 & 0.53 \\
\hline TCT Group & 0.010 & 0.048 & 0.20 & 0.192 & 0.124 & 1.54 & 0.555 & 2.506 & 0.22 & 1.204 & 7.230 & 0.17 \\
\hline 1-Hr Follow Up & -0.038 & 0.022 & -1.74 & -0.035 & 0.036 & -0.97 & 1.803 & 1.067 & 1.69 & 5.062 & 3.297 & 1.54 \\
\hline 12-Week Follow Up & 0.004 & 0.024 & 0.18 & -0.054 & 0.039 & -1.40 & -0.699 & 1.139 & -0.61 & -3.367 & 3.516 & -0.96 \\
\hline SIN & -0.002 & 0.001 & $-2.15^{*}$ & 0.000 & 0.003 & -0.17 & 0.077 & 0.054 & 1.45 & 0.251 & 0.154 & 1.63 \\
\hline TCT $\times 1-\mathrm{Hr}$ & 0.046 & 0.045 & 1.03 & -0.061 & 0.073 & -0.83 & -2.245 & 2.160 & -1.04 & -3.939 & 6.668 & -0.59 \\
\hline TCT x 12-Week & 0.025 & 0.062 & 0.40 & -0.070 & 0.103 & -0.68 & -2.458 & 3.002 & -0.82 & -6.153 & 9.227 & -0.67 \\
\hline 1-Hr x SIN & 0.002 & 0.001 & 1.60 & 0.002 & 0.002 & 1.36 & -0.064 & 0.046 & -1.41 & -0.177 & 0.141 & -1.26 \\
\hline 12-Week x SIN & 0.000 & 0.001 & 0.27 & 0.003 & 0.002 & 1.56 & 0.016 & 0.048 & 0.34 & 0.086 & 0.149 & 0.58 \\
\hline TCT x 1-Hr x SIN & -0.002 & 0.002 & -0.83 & 0.002 & 0.003 & 0.62 & 0.069 & 0.090 & 0.76 & 0.090 & 0.279 & 0.32 \\
\hline TCT x 12-Week x SIN & -0.001 & 0.003 & -0.50 & 0.003 & 0.004 & 0.61 & 0.110 & 0.123 & 0.89 & 0.278 & 0.379 & 0.73 \\
\hline
\end{tabular}

Note: $\mathrm{RMS}=$ root mean square; $\mathrm{SNR}=$ signal-to-noise ratio; $\mathrm{dB}=$ decibel 


\section{Figure Captions}

Figure 1. Grand average waveforms for the treatment as usual group (top) and targeted cognitive training group (bottom) at recording site $\mathrm{Cz}$.

Figure 2. Estimated marginal means with their respective $95 \%$ confidence intervals for each group and time point. $\mathrm{RMS}=$ root mean square. $\mathrm{SNR}=$ signal-to-noise ratio.

Figure 3. A) The relationship between scores on the $4 \mathrm{~dB}$ level of the Words-in-Noise Test (WIN) and predicted post-stimulus root mean square (RMS) amplitude shown separately for each group. B) The relationship between scores from the Quick Speech-in-Noise Test (SIN) and predicted pre-stimulus RMS amplitude is shown. C) The predicted relationship between scores from the SIN and predicted dB signal-to-noise ratios (SNRs) is shown. Shaded regions represent the $95 \%$ confidence intervals. 
Figure 1
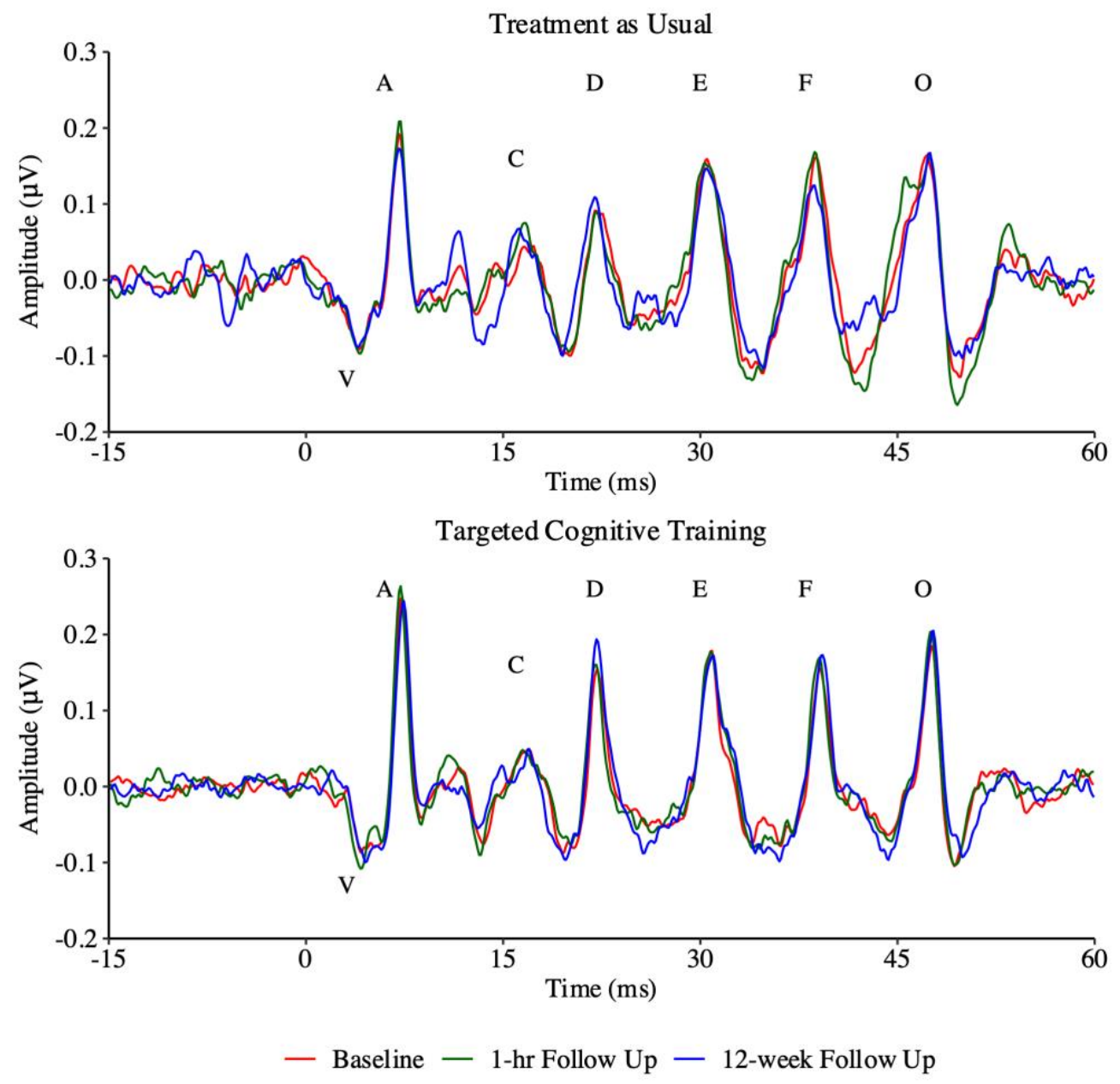
Figure 2
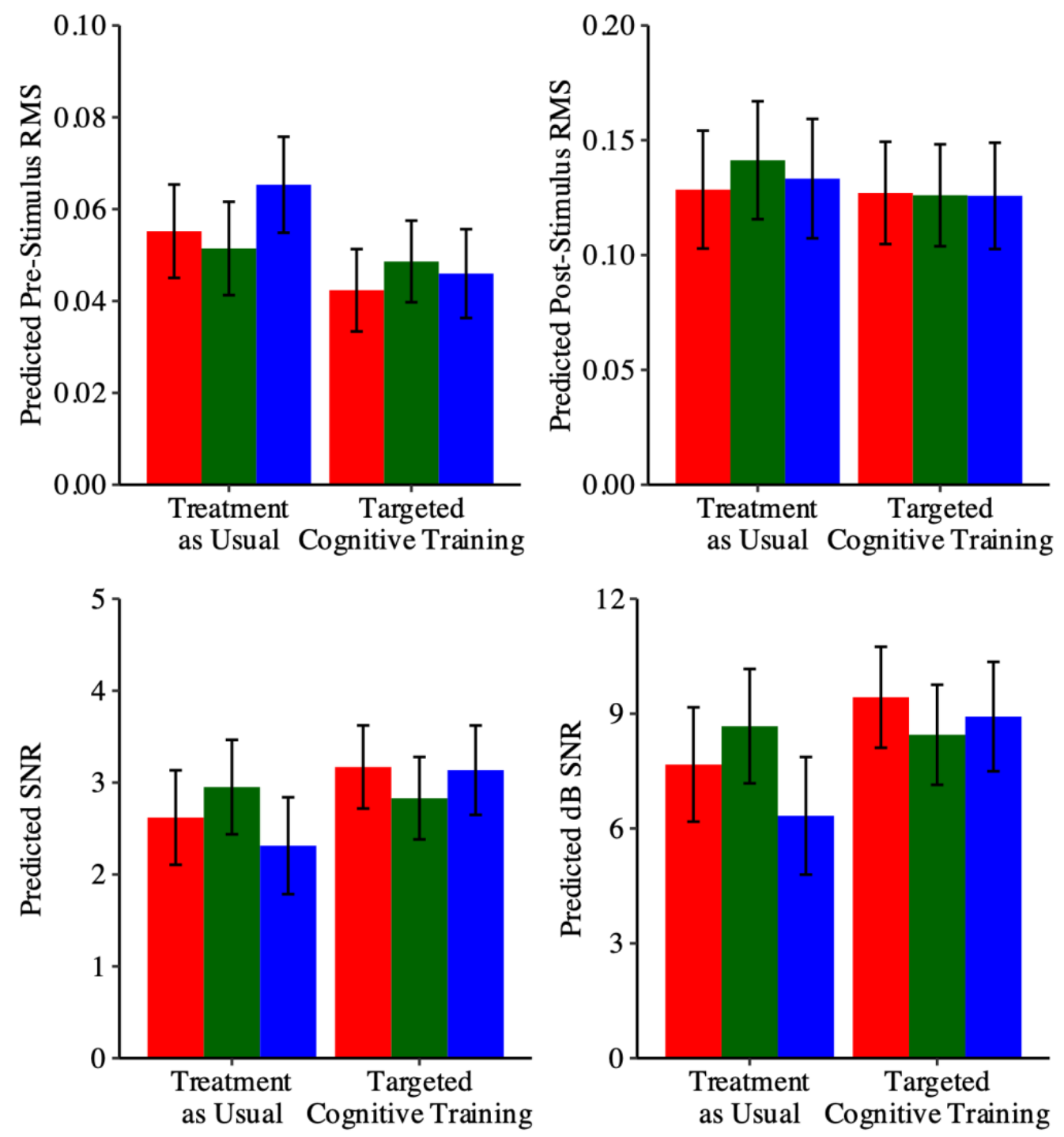

Baseline 1-hr Follow Up 12-week Follow Up 
Figure 3
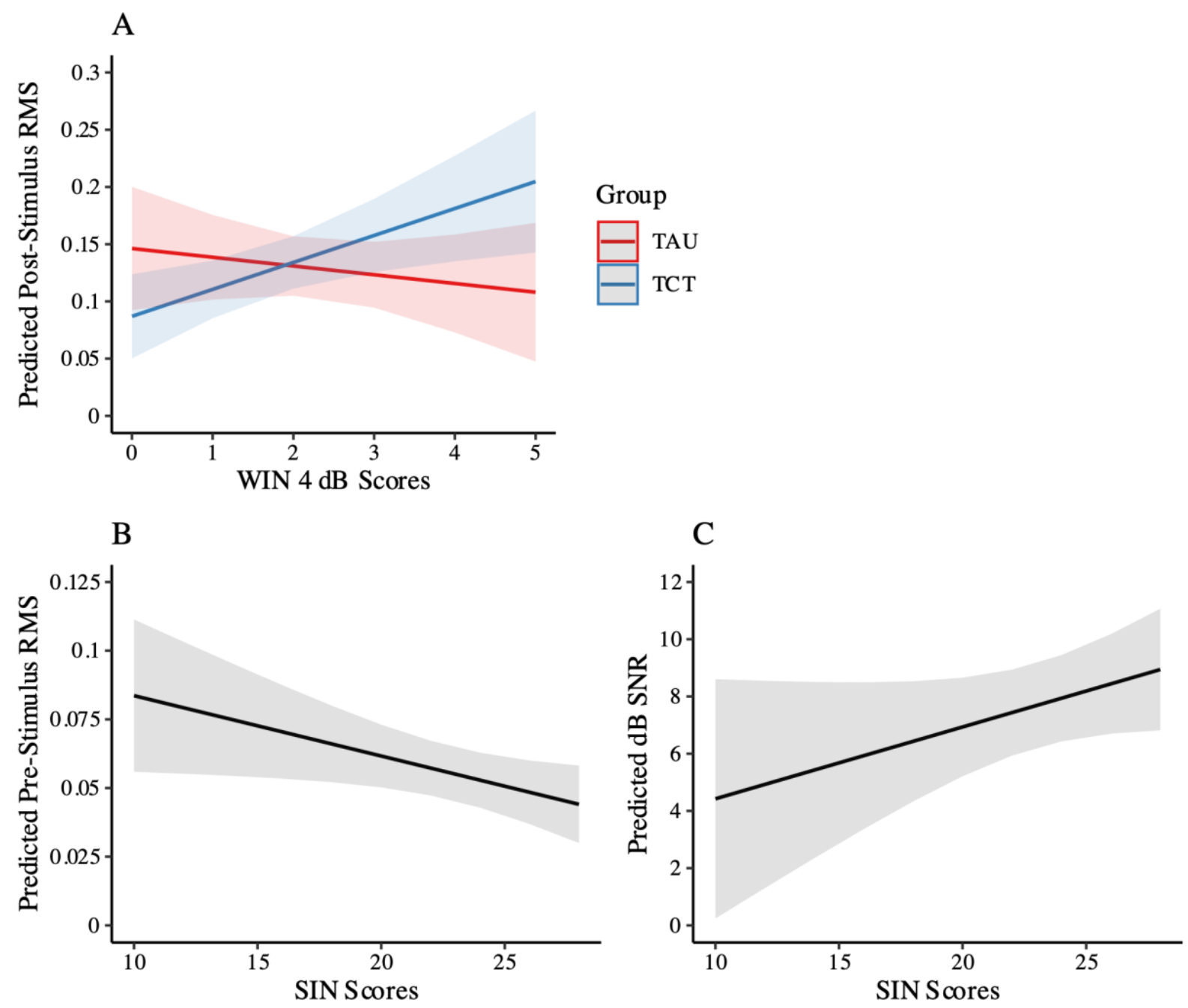